Poster (C13)

Cervical Cancer

https://doi.org/10.3802/jgo.2021.32.S1.C13

\section{Treatment outcomes in early stage cervical cancer: experience of a gynecological oncology unit from India}

\section{Hemlata Garg, " Megha Nandwani, Satinder Kaur}

Dharamshila Narayana Hospital, New Delhi, India (garg.hema@gmail.com)

Objective: To analyze and critically evaluate the clinicopathological features and survival outcomes of early stage cancer cervix patients and to reclassify all histopathology reports according to new International Federation of Gynecology and Obstetrics (FIGO) 2018 staging classification and compare their survival outcome.

Methods: A retrospective observational analysis was done from 1st June 2013 to 31st May 2018; over a period of 5 years for patients who underwent radical hysterectomy followed by risk-based adjuvant therapy in our center and later patients were analyzed and evaluated for clinic-pathological features and survival outcome. Patients were reclassified as per histopathology reports according to new FIGO 2018 and their survival outcome were compared with old FIGO staging. Results: The 100 patients of early-stage cancer cervix were studied. All patients underwent open radical hysterectomy with bilateral pelvic lymph node dissection. The median age of the study population was 52.5 years with median follow up of 62.1 months. The overall survival and relapse free survival were $87.5 \%$ and $91.4 \%$, respectively. The study population was then reclassified according to new FIGO 2018 staging. It was seen that the patients with stage IB1 and IB2 cervical cancer had overall survival of $91.1 \%$ and $90 \%$, respectively. The overall survival of stage IB3 was $80 \%$ and the survival of stage IIIC1 was only $60 \%$.

Conclusion: Early stage cancer cervix have good overall survival outcome when treated appropriately with surgery and risk based tailored therapy.

Poster (C14)

Cervical Cancer

https://doi.org/10.3802/jgo.2021.32.S1.C14

\section{After the laparoscopic approach to cervical cancer (LACC) trial: minimally invasive surgery for early cervical cancer}

Min Jeong Park, Shin-Wha Lee, Jeong-Yeol Park, Dae-Yeon Kim, " Dae-Shik Suh, Yong-Man Kim, Young-Tak Kim Asan Medical Center, Seoul, Korea (kdyog@amc.seoul.kr)
Objective: The aim of this study was to compare minimally invasive surgery (MIS) with open abdominal radical hysterectomy (RH) in patients with early-stage cervical cancer after major prospective study called laparoscopic approach to cervical cancer trial in November 2018.

Methods: A retrospective data analysis was performed on patients who underwent surgery at our hospital for early-stage cervical cancer from January 2019 to September 2020. Surgery was compared with classical RH and MIS (laparoscopic, robotic). The patient's stage was IA2-IB2 based on the 2018 International Federation of Gynecology and Obstetrics stage, and the pathology was squamous cell carcinoma, adenocarcinoma, and adenosquamous type.

Results: A total of 149 patients with stage IA2-IB2 cervical cancer were analyzed retrospectively. The 76 patients (48.1\%) received MIS (laparoscopic: 56, robotic: 20) and 82 patients (51.9\%) underwent open RH surgery. Median follow-up was 23.5 months (range: 13-33). One person who received laparoscopic RH out of a total of 149 people recurred.

Complications occurred in 1 case during surgery and 4 case after surgery for open $\mathrm{RH}, 0$ during surgery and 2 after surgery for MIS. There were no other significant differences in the amount of bleeding or length of hospital stay.

Conclusion: Despite of short-term follow up period in our data, there was no difference in the oncological outcome between the 2 groups. Microinvasive surgery can be considered through sufficient discussion with the patient in the case of early stage.

Poster (C15)

Cervical Cancer

https://doi.org/10.3802/jgo.2021.32.S1.C15

\section{Association of pattern-based classification system to survival outcome in women with cervical adenocarcinoma}

\author{
Natcha Jantarateptewan, " Marut Yanaranop, \\ Sakchai Jitpakdee \\ Rajavithi Hospital, Bangkok, Thailand (natcha.j24@gmail.com)
}

Objective: To assess the survival outcome and recurrence according to pattern-based classification system of cervical adenocarcinoma.

Methods: A retrospective cohort study was conducted in women pathologically diagnosed of endocervical adenocarcinoma (ECA) underwent primary surgery at Rajavithi Hospital during January 2012 and December 2017. Post-operative pathologic slides were reviewed according to the pattern-based classification system. Survival outcome and recurrence were 